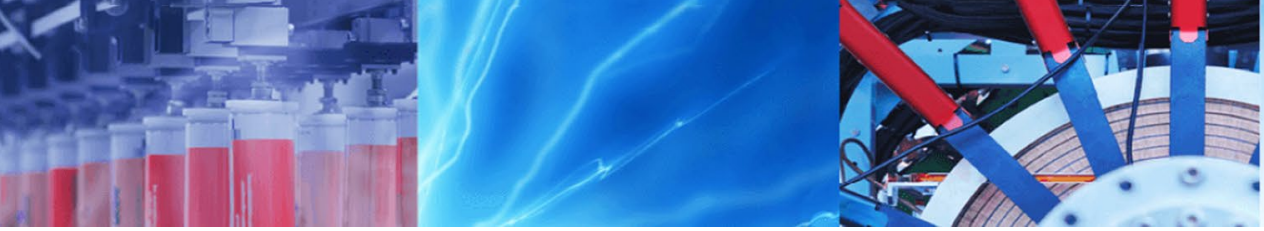

Research Article

\title{
Improvement in mechanical and structural properties of poly(ethylene terephthalate) nanohybrid
}

\author{
Dipti Saxena ${ }^{1} \cdot$ Dipak Rana $^{2} \cdot$ E. Bhoje Gowd ${ }^{3} \cdot$ Pralay Maiti $^{1}$ (I)
}

(c) Springer Nature Switzerland AG 2019

\begin{abstract}
The wide range of applicability of poly(ethylene terephthalate) (PET) in various fields such as packaging, automobile parts, sports and textile due to its transparent nature, light weight and mechanical properties creates strong case to enhance its mechanical properties. By embedding nanofillers and thereby improving the mechanical properties, this spectrum can further be widened. The effect of addition of nanofiller in terms of stress concentration is a crucial phenomenon to understand the mechanical property enhancement. The nanohybrids of poly(ethylene terephthalate) (PET) and organically modified nanoclay have been prepared through solution casting route to enhance the properties significantly without any trade-off of deteriorating other properties. The dispersion of nanoclays in the polymer matrix has been observed through TEM images. Good level of dispersion has been achieved in nanohybrids due to specific interactions between nanoclay and PET matrix explored through XRD and FTIR spectroscopic measurements. Thermal stability of the nanohybrids has been tested through TGA and DSC analyses. The mechanical properties have been tested and found to be enhanced in the presence of nanoclay with increasing filler concentrations. The modulus has been increased for nanohybrids up to $93 \%$, while the optimum value of modulus and toughness has been observed at $4 \mathrm{wt} \%$ filler percentage. The improvement in mechanical properties has been predicted using different models for randomly dispersed filler and has been found to be fitted in acceptable range. The hardness of the nanohybrids has been tested using Vickers hardness test and is found to be increasing continuously with filler loading. The structural formations upon uniaxial stretching have been contemplated through two-dimensional small-angle X-ray scattering as well as through wide-angle XRD. The nanohybrids have shown short-range ordering on uniaxial stretching in comparison with pristine PET. Blob size has been found out using Debye-Bueche model and is found to be increased upon stretching the samples. The polymer nanohybrids have been prepared for the mechanical property improvement without imparting high brittleness for practical applications. The behavior of nanoclay on stretching the polymer nanohybrids has also been studied in detail.
\end{abstract}

Keywords Poly(ethylene terephthalate) $\cdot$ Nanohybrid $\cdot$ Mechanical properties $\cdot$ Vickers hardness $\cdot$ Small-angle X-ray . Stretching effect

Electronic supplementary material The online version of this article (https://doi.org/10.1007/s42452-019-1406-3) contains supplementary material, which is available to authorized users.

Pralay Maiti, pmaiti.mst@itbhu.ac.in | ${ }^{1}$ School of Materials Science and Technology, Indian Institute of Technology (Banaras Hindu University), Varanasi 221005, India. ${ }^{2}$ Industrial Membrane Research Institute, Department of Chemical and Biological Engineering, University of Ottawa, 161 Louis Pasteur St., Ottawa, ON KIN 6N5, Canada. ${ }^{3}$ Materials Science and Technology Division, CSIR-National Institute for Interdisciplinary Science and Technology, Trivandrum, Kerala 695019, India. 


\section{Introduction}

Various methods have been used in the past to enhance PET's properties making it usable for superior applications. Various blends of PET have been made to alter its properties, but the enhancement in properties is not significant considering its energy consuming process [1]. The polymer composites are prepared by inducing fillers into the polymer matrix and have been widely used to enhance the material properties as it needs very little amount of filler. Polymer nanocomposites make use of nanofillers which give enormous increase in the material property due to its large surface area available to interact with polymer matrix. By dispersing various nanofiller in the polymer matrix, the material can obtain biocompatibility, mechanical strength, thermal stability, conductivity, flame retardancy, etc. [2-6].Various fillers such as montmorillonite [7], Cloisite 15A, Nanolin DK2 (CEC 115-120 mequiv. per $100 \mathrm{~g}$ and intergallery spacing of $2.4 \mathrm{~nm}$ ) [8], layered double hydroxides [9], silica [10], fluoromica (2:1 type layered silicate with cation exchange capacity of $70 \mathrm{meq} / 100 \mathrm{~g}$ and intergallery spacing of $0.95 \mathrm{~nm})[11,12]$ and phosphate glass [13] have been used with the polymer matrix to enhance the material properties. Montmorillonite clay has widely been used to enhance the properties of a wide range of polymers. It has a 2:1 layered structure where twodimensional single layer of aluminum octahedral layer is sandwiched between two tetrahedral layers of silicon tetrahedron oxide $\left(\mathrm{SiO}_{4}\right)$ (silicon atom bonded with four oxygen atoms). Cloisite 15A, Nanolin, layered double hydroxides, fluoromica are the clays which have similar layered structures modified in different ways having different intergallery spacings and cation exchange capacities. Montmorillonite clay (Cloisite 30B) has previously been used to enhance the properties of nanocomposites $[7,14,15]$. It is natural montmorillonite clay which is modified with methyl tallow bis-2-hydroxyethyl quaternary ammonium ion. Ghasemi et al. [14] prepared PET/ clay nanocomposites prepared through melt casting method and showed meager $20 \%$ increment in tensile modulus. The presence of clay induced the increase in modulus, whereas the severe brittleness was associated with this. Crystallization behavior in the presence of clay was also observed through wide-angle XRD. Scaffaro et al. [16] prepared PET nanocomposites using two different organically modified nanoclays, namely Cloisite $15 \mathrm{~A}$ and 30B. The melt compounding route was opted to prepare those nanocomposites. Mechanical properties and thermal stability were studied with varying filler concentrations. Young's modulus was not significantly improved until at higher weight percentage of filler concentration ( $10 \mathrm{wt} \%)$, although the elongation at break dropped drastically even at lower filler content ( 3 $w t \%)$. The thermal stability of the nanocomposites was also reduced as measured through melt rheology and intrinsic viscosity measurements. Ghanbari et al. [15] prepared PET nanocomposites using two organically modified clay (30B and $\mathrm{N} 28 \mathrm{E}$ ) using melt blending in the presence of multifunctional epoxy-based chain extender. Young's modulus was improved up to $33 \%$ using $4 \mathrm{wt} \%$ 30B clay, whereas reduction in toughness was around $92 \%$. Yang et al. [17] reported supercritical carbon dioxide pre-dispersed Cloisite 30B melt extruded with PET matrix. The Young's modulus and tensile strength were improved by $12 \%$ and $\sim 25 \%$, respectively, whereas elongation at break was reduced by $\sim 80 \%$. As obvious, there is a need to improve the mechanical strength keeping its toughness intact.

In this work, nanohybrids of PET have been prepared using Cloisite $30 \mathrm{~B}$ through solution casting route. The dispersion of clay in the PET matrix, clue to generate better mechanical strength, has been studied in detail. The hardness of the nanohybrids has been modeled as measured through Vickers hardness test which, to our best knowledge, has not been worked out previously. The structural advancement in the nanohybrids has been examined by SAXS (small-angle X-ray scattering), and the effect of stretching on PET nanohybrids has been studied in detail.

\section{Experimental}

\subsection{Materials}

Poly(ethylene terephthalate) granules were obtained from Otto Chemie Pvt. Ltd., India. Cloisite 30B clay (natural montmorillonite organically modified with methyl tallow bis-2-hydroxyethyl quaternary ammonium) was used as filler material.

\subsection{Preparation of nanohybrids}

Solution casting route was used to prepare PET nanohybrid. The pristine PET was dissolved in dichloromethane (DCM) by stirring it for $40 \mathrm{~min}$ at room temperature. 30B nanoclay was sonicated in DCM separately in a beaker for $40 \mathrm{~min}$ to disperse the clay particles evenly and to diminish the agglomeration of nanoclay particles. The pure PET and the dispersed clay solution were then mixed. This mixture was stirred for an hour for the proper dispersion of the clay into the polymer. After that the solution was poured into a Petri dish and dried for $15 \mathrm{~h}$ which was further dried for $24 \mathrm{~h}$ in vacuum at $50^{\circ} \mathrm{C}$ for the solvent to be completely evaporated. 


\subsection{Sample preparation}

The dog bone-shaped samples were prepared using injection molding technique (Haake micro-injector). The nanohybrids were heated at their melting temperature around $250^{\circ} \mathrm{C}$. The mold temperature was kept at $40^{\circ} \mathrm{C}$. The samples were prepared of the dimension: gauge length $=20 \mathrm{~mm}$, width $=4 \mathrm{~mm}$ and thickness $=2.14 \mathrm{~mm}$. The pressure for microinjection was applied as 100 bar.

\section{Characterization}

\subsection{Transmission electron microscopy (TEM)}

TEM (FEI Technai 20) was used at an accelerating voltage of $200 \mathrm{kV}$ for the observation of the dispersion of nanoclay in the polymer matrix. Samples were cut of ultrathin sections using Leica ultracut UCT equipped with a diamond knife at $-80^{\circ} \mathrm{C}$.

\subsection{Scanning electron microscopy (SEM)}

Morphological investigation of pure PET and PET nanohybrids was done through scanning electron micrographs using SEM (SUPRA 40, Zeiss). The gold coating of the samples was done prior to experiment using sputtering apparatus.

\subsection{X-ray diffraction}

The intercalation or exfoliation of clay is an essential parameter for nanohybrid. This was examined using X-ray diffraction (XRD) technique. Rigaku Miniflex 600 X-ray diffractometer was used to investigate the diffraction pattern. The samples were scanned at room temperature at a voltage of $45 \mathrm{kV}$ with CuKa radiation $(\lambda=1.54 \AA$ ). Diffraction angle $(2 \theta)$ range was kept from $1^{\circ}$ to $40^{\circ}$ with a scan rate as $1 \% \mathrm{~min}$.

\subsection{Fourier-transform infrared spectroscopy (FTIR)}

FTIR spectra were achieved using Nicolet 5700 instrument, and the range was taken from 650 to $3500 \mathrm{~cm}^{-1}$. The measurements were performed in transmittance mode at room temperature having a resolution of $4 \mathrm{~cm}^{-1}$. Approximately $100-\mu \mathrm{m}$-thick samples were used.

\subsection{Thermogravimetric analysis (TGA)}

Degradation temperature and thermal stability of PET and its nanohybrids were examined using TGA. It was investigated using a thermogravimetric analyzer of Mettler
Toledo which was equipped with a differential thermal analyzer. The samples were put in an alumina crucible. Heating rate was taken as $20 \% \mathrm{~min}$ under nitrogen atmosphere. All the data were taken in a temperature range of $30-600^{\circ} \mathrm{C}$.

\subsection{Differential scanning calorimetry (DSC)}

DSC experiment was done to observe the glass transition temperatures of amorphous PET and its nanohybrid films using Mettler 832. The calibration was done with indium and zinc ahead of the experiment. The samples were kept in an aluminum pan. The temperature range was kept as $25-300^{\circ} \mathrm{C}$ at a scan rate of $10^{\circ} / \mathrm{min}$. The weight of the sample was in the range from 4 to $10 \mathrm{mg}$.

\subsection{Mechanical properties}

Tensile testing measurements were performed at room temperature on Instron Universal Testing Machine (Instron 3369 , load cell of $50 \mathrm{kN}$ capacity). The modulus of elasticity $(E)$ and toughness were evaluated from the graph. The injection-molded dog bone samples were used to be stretched up to the fracture point. Cross-head speed was taken as $5 \mathrm{~mm} / \mathrm{min}$, and the experiments were performed at room temperature. To ensure minimum error estimation, five samples of each nanohybrid were tested.

\subsection{Hardness test}

Vickers hardness tester (Tinius Olsen, FH5 series, Indenter no. DKD4132) was used to evaluate the microhardness of different samples. The indenter of the instrument had a square-based pyramid indenter having $136^{\circ}$ angle between its opposite faces. $0.5 \mathrm{kgf}$ was applied for a dwell period of $15 \mathrm{~s}$ on each sample. Two diagonals of the indentation obtained were measured, and their average was calculated. For each sample, at least 10 measurements were done and the average values have been reported. The microhardness tester reveals the hardness in the form of VHN (Vickers hardness number) which is defined as:

$\mathrm{VHN}=\frac{2 F \sin \left(\frac{136^{\circ}}{2}\right)}{d^{2}}=1.854 \frac{F}{d^{2}}$

where $F$ is force in kgf, $d$ is the mean of the diagonals resulted from the indentation.

\subsection{Small-angle X-ray scattering}

SAXS measurements were performed to determine the effect of orientation/local ordering by stretching of PET 
and its nanohybrid. The system consisted of a $6 \mathrm{~kW}$ rotating anode generator (XEUSS 2D SAXS) operated at $50 \mathrm{kV}$ and $0.6 \mathrm{~mA}$ with CuKa, radiation and Ge monochromator. The data were analyzed for $6 \mathrm{~h}$. 2D SAXS images were azimuthally integrated to obtain 1D scattering intensity profiles as a function of $q$ (magnitude of scattering vector), $q=4 \pi \sin \theta / \lambda$, where $2 \theta$ is the scattering angle. The obtained scattering intensity SAXS profiles were corrected for background scattering.

\section{Results and discussion}

\subsection{Dispersion and interactions}

The prediction about the nanoclay dispersion in PET matrix can be deduced from conclusive TEM and XRD results. The dispersion of nanoclays in polymer matrix has been compared through TEM analysis. The TEM images, shown in Fig. 1a, describe the dispersion of the nanoclay in the PET matrix. The 30B clay platelets have been dispersed in the polymer matrix uniformly as can be seen in the low magnification TEM image of Fig. 1 a. The intercalated and some exfoliated morphology can be seen in the high magnification image of Fig. 1a; inset image shows the increased intergallery spacing due to intercalation of polymer chains between clay platelets. The clay platelets have average aspect ratio (ratio of length and thickness of nanoparticle aggregate) of $\sim 20$

(a)
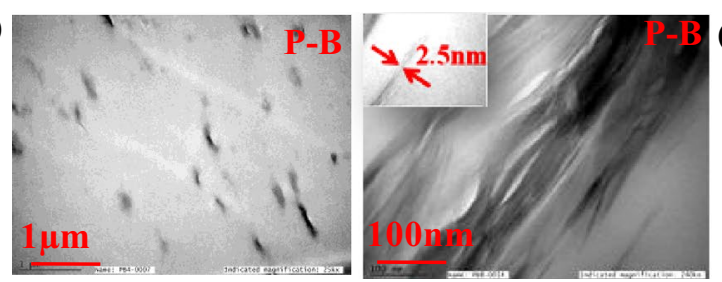

(b)

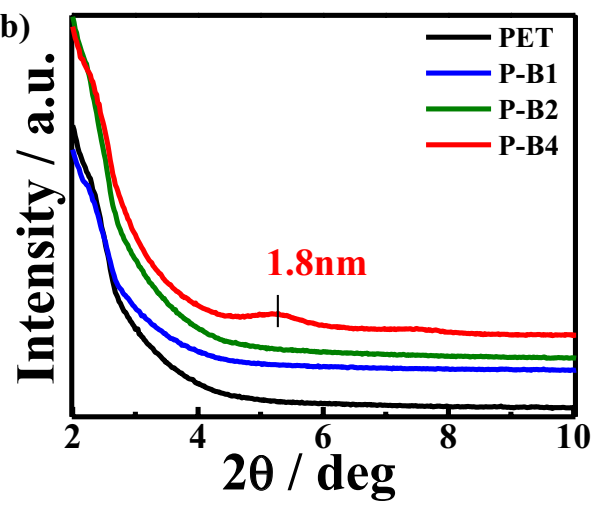

Fig. 1 a TEM images of the P-B nanohybrid at low (left) and high magnification (right); inset figure shows the intercalation; $\mathbf{b}$ XRD spectra of pure PET and P-B at different nanoclay concentrations; $c$ and average correlation length (i.e., distance between two nanoparticles) $[5,18]$ of $430 \mathrm{~nm}$. Hence, the TEM images show good level of intercalation and some exfoliation of the nanoclay platelets in the PET nanohybrids.

The XRD patterns of the nanohybrids are shown in Fig. 1b. For the lower percentage of nanoclay inclusion, there is no clear peak to identify d-spacing. The absence of well-defined peaks could be due to the random orientation/disordered structure of the nanoclay in the polymer matrix. This signifies that there is very less ordering of the clay platelets distribution in the polymer matrix. The peak at $1.8 \mathrm{~nm}$ could be due to the fact that the nanohybrids have some unintercalated structure or there is structure formation in the nanohybrid. There exist different kinds of tactoids which are not perfectly ordered and are having different interlayer distances resulting less coherency. However, the clear intercalation can be seen in the TEM images (inset of Fig. 1a), whereas it is not so evident in XRD spectra presumably because of the disordered structure. SEM images of the nanohybrids are shown in Fig. 1c. The morphology of nanohybrids shows the presence of nanoclay in PET matrix. The surface roughness has increased in the presence of nanoclay. FTIR spectroscopy has been performed to check the interactions of nanoclay and PET. The carbonyl $(C=O)$ peak has slightly been shifted from 1706 to $1708 \mathrm{~cm}^{-1}$ in the nanohybrid $[19,20]$. The peak at $709 \mathrm{~cm}^{-1}$, due to ring $\mathrm{C}-\mathrm{C}$ bending and ring $\mathrm{C}-\mathrm{H}$ out of plane stretching,
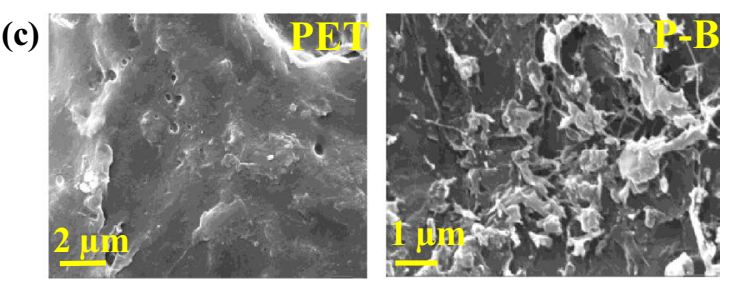

(d)

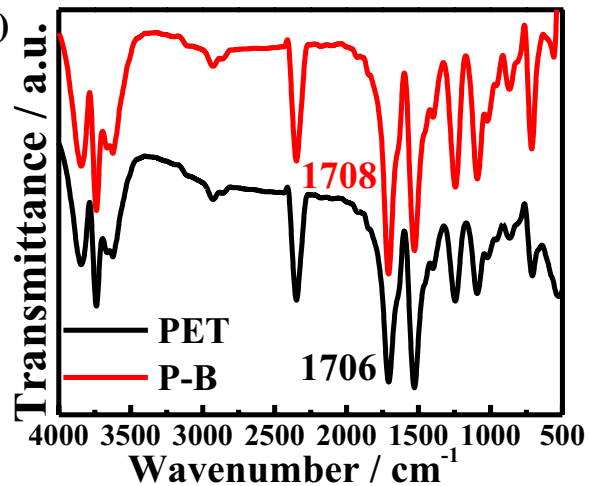

SEM images of pure PET and P-B showing the surface morphology; and d FTIR spectra showing interactions in nanohybrid 
has been shifted to $713 \mathrm{~cm}^{-1}$ [21]. This shifting of peaks is due to the interactions of clay with polymer matrix.

\subsection{Thermal properties and stability}

Thermal properties of nanohybrids are shown in Fig. 2a, b. The TGA thermograms in Fig. 2a show that there is very slight reduction in degradation temperature of $\mathrm{P}-\mathrm{B}$ nanohybrid (the degradation temperature has been taken at temperature corresponding to $5 \%$ weight loss). The degradation temperature of $\mathrm{P}-\mathrm{B}$ has come out to be $408^{\circ} \mathrm{C}$, whereas the degradation temperature of pure PET has been found to be $410^{\circ} \mathrm{C}$. The mere fall in degradation temperature is due to the collapse of the clay platelets because of the degradation of the modifier present in the nanoclay gallery at higher temperature during processing the film. This is to mention that the degradation of 30B starts around $182{ }^{\circ} \mathrm{C}$ [16]. The glass transition temperature is shown both through DTA and DSC thermograms in Fig. 4a (inset), $b$, respectively. The glass transition temperature has been found out to be the same, i.e., $65^{\circ} \mathrm{C}$ and there is no reduction/increase in glass transition temperature in presence of nanoclay.

\subsection{Mechanical responses and predictions}

The mechanical properties of the nanohybrids are shown in Fig. 3. The stress-strain curves of P-B are shown in Fig. 3a. The modulus of nanohybrids is shown in Fig. 3b. The modulus has been increasing with the increment of the nanoclay content. The toughness is shown in Fig. 3c. The 30B clay induces significant increase in Young's modulus (93\%) for $8 w t \%$ of nanoclay inclusion. It can be seen that the toughness of the nanohybrids has been retained up to $4 \mathrm{wt} \%$ of clay concentration. The previous studies have reported huge toughness reductions more than $90 \%$ at $4 \mathrm{wt} \%$ of clay loadings $[15,16]$. In this study, the toughness reduction is merely $12 \%$ for $4 \mathrm{wt} \%$ of filler concentration. However, the increasing amount of $30 \mathrm{~B}$ is inducing brittleness. The elongation at break is reducing at $8 \mathrm{wt} \%$ of $30 \mathrm{~B}$ inclusion. The increase in modulus for $8 \mathrm{wt} \%$ nanoclay concentration has been consolidated with the increased brittleness. The increase in brittleness with higher $30 \mathrm{~B}$ concentration has been reported previously [15]. The toughness in P-B nanohybrids has been reduced for $8 \mathrm{wt} \%$ nanoclay which could be attributed to the agglomeration of the clay particles as well as interfacial debonding which results in voids and flaws in the matrix causing early failure of the material. However, previously reported results showed that the toughness reduced by more than $90 \%$ at lower percentage of clay [15]. This sudden decrement at even lower percentage of clay was possible because of melt extrusion process, where nanoclay degraded to a greater extent. In this work, solution casting route has been adopted in which the processing temperature has not been that high. Hence, the clay degradation has not been occurred causing considerably high modulus and very low reduction in toughness at $4 \mathrm{wt} \%$ nanoclay (P-B4). The toughness reduction for $4 \mathrm{wt} \%$ of nanoclay inclusion has been mere $12 \%$. The $4 \mathrm{wt} \%$ nanoclay inclusion has been the optimum where the modulus increment of $21 \%$ and toughness reduction only $12 \%$ has been achieved. This balance of increment of modulus retaining toughness is better than previously reported studies [14-16]. The values of modulus for different filler concentrations have been predicted by different micromechanical models.

\subsubsection{Halpin-Tsai model}

Halpin-Tsai model is widely used for the modulus prediction of composites. This model takes into account the large spectrum of filler geometry as well as orientations. The filler geometries include fiber, flake as well as plate-like geometries having continuous or discontinuous orientations. The Young's modulus is predicted by $[22,23]$ :
Fig. 2 a TGA thermograms of pure PET and P-B nanohybrids; inset figure shows the glass transition temperature through DTA thermograms; and $\mathbf{b}$ DSC thermograms showing glass transition temperature of pristine PET and P-B nanohybrid
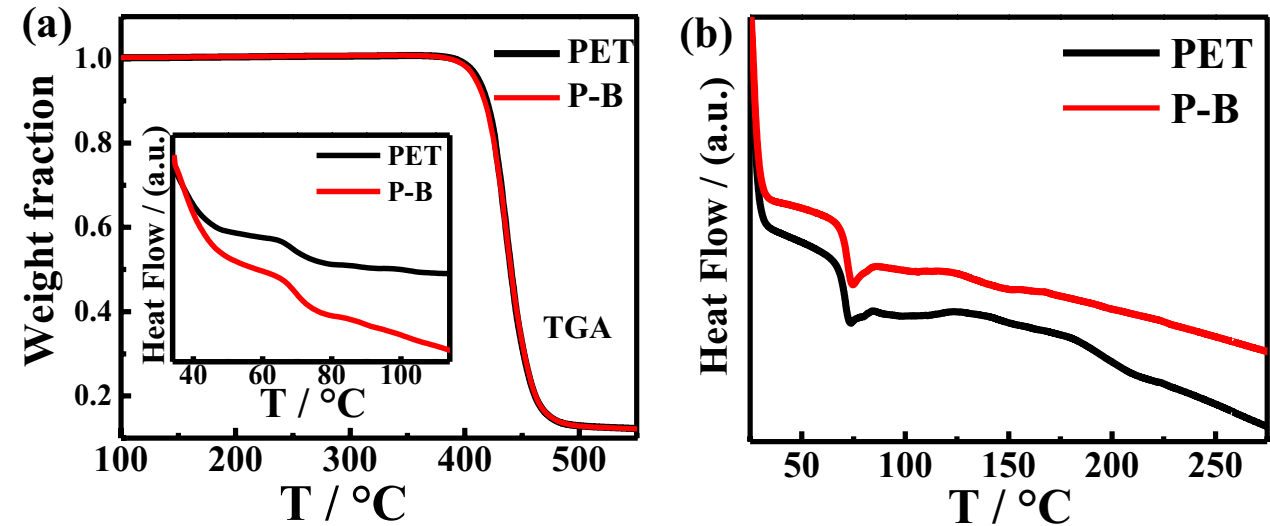

SN Applied Sciences A SPRINGER NATURE journal 


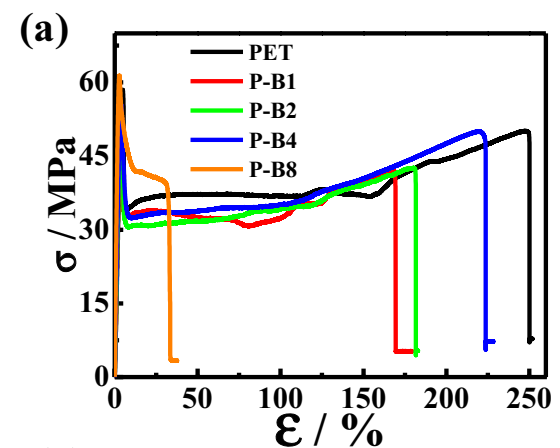

(d)

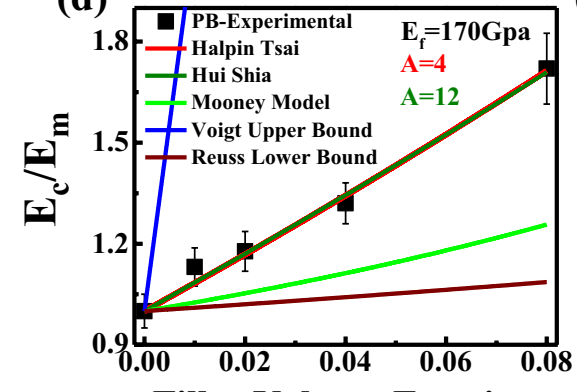

Filler Volume Fraction (b)

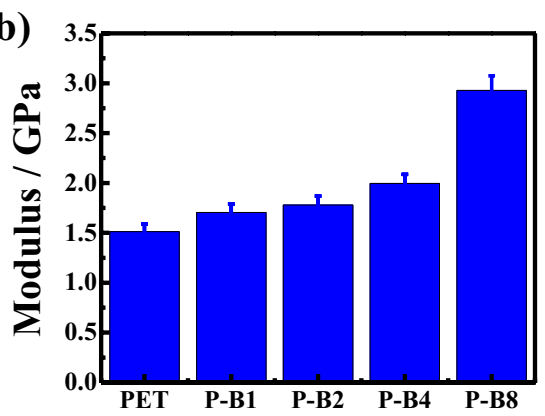

(e)

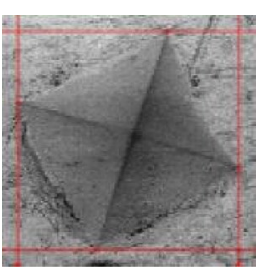

PET

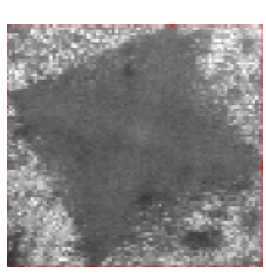

P-B (c)

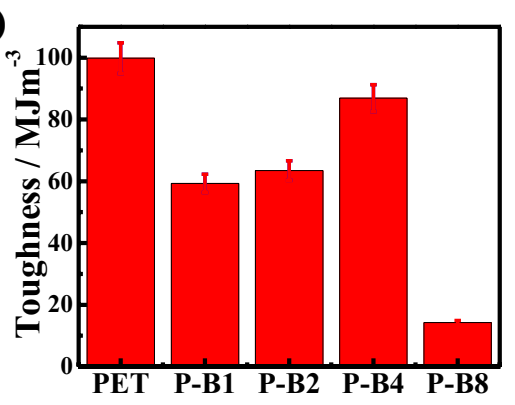

(f)

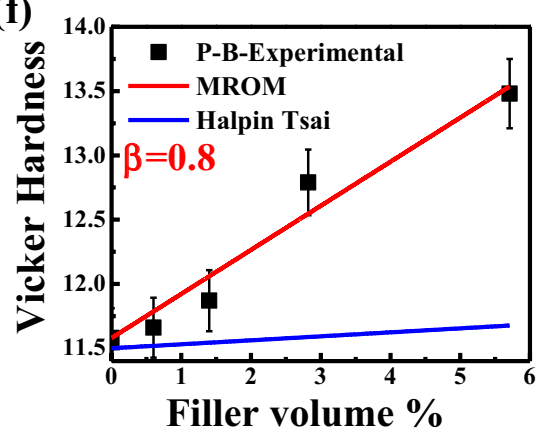

Fig. 3 a Stress-strain curves of nanohybrids at different indicated concentrations comparing pure PET; $\mathbf{b}$ elastic modulus values of indicated nanohybrids at different filler concentrations as calculated from the stress-strain curve; $c$ toughness values of the nanohybrids at different filler concentrations as calculated from the area under stress-strain curves; $\mathbf{d}$ prediction of elastic modulus values using different micromechanical models; e Vickers hardness test imprints on the pure PET and P-B nanohybrid; and $\mathbf{f}$ prediction of hardness values using different models as discussed in the main text
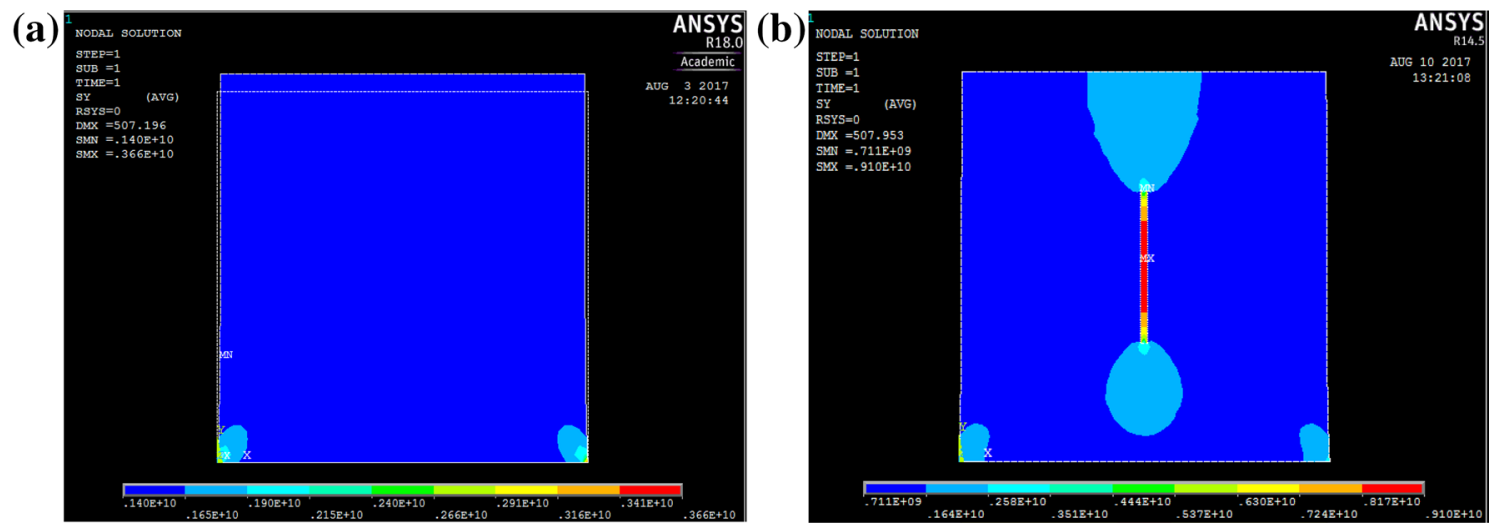

Fig. 4 a Stress distribution in pure PET; and $\mathbf{b}$ stress distribution in PET nanohybrid as obtained using ANSYS software

$\frac{E_{c}}{E_{m}}=\frac{1+\zeta \eta \phi_{f}}{1-\eta \phi_{f}}$

$\eta=\frac{\frac{E_{f}}{E_{m}}-1}{\frac{E_{f}}{E_{m}}+\zeta}$ where $E_{c}, E_{m}$ and $E_{f}$ are the Young's modulus of composite, matrix and filler, respectively. $\phi_{f}$ is the filler volume fraction. $\zeta$ is the shape parameter which is dependent on filler geometry as well as loading direction and defined as $\zeta=2 A=2(I / d)$ for disk-like and $2(/ / t)$ for plate-like geometry. $A$ is aspect ratio, and $I, d, t$ are the length, diameter and thickness, respectively. 


\subsubsection{Hui-Shia model}

The Hui-Shia model predicts the elastic modulus taking the assumption of perfect interfacial bonding between filler and the matrix and unidirectional filler alignment. The model is proposed as [23]:

$\frac{E_{c}}{E_{m}}=\frac{1}{1-\frac{\phi_{f}}{4}\left[\frac{1}{\xi}+\frac{3}{\xi+\Lambda}\right]}$

$\xi=\phi_{f}+\frac{E_{m}}{E_{f}-E_{m}}+3\left(1-\phi_{f}\right)\left[\frac{(1-g) \alpha^{2}-\frac{g}{2}}{\alpha^{2}-1}\right]$

$g=\frac{\pi}{2} \alpha$

$\bigwedge=\left(1-\phi_{f}\right)\left[\frac{3\left(\alpha^{2}+0.25\right) g-2 \alpha^{2}}{\alpha^{2}-1}\right]$

where $a$ is inverse aspect ratio $(a=1 / A)$.

\subsubsection{Voigt upper bound and Reuss lower bound model}

Halpin-Tsai model gives the maximum value when $\zeta$ reaches infinity which is called Voigt rule of mixtures, where matrix and fiber reach same uniform strain, called isostrain approach. For the upper bound value of elastic modulus, $\zeta$ is replaced by infinity and given as:

$E_{c}=\phi_{f} E_{f}+\left(1-\phi_{f}\right) E_{m}$

when the minimum value of the Young's modulus is to be calculated, the $\zeta$ reaches to 0 and is given as Reuss inverse rule of mixtures:

$\frac{1}{E_{c}}=\frac{\phi_{f}}{E_{f}}+\frac{\left(1-\phi_{f}\right)}{E_{m}}$

Both these models give the upper and lower ranges of elastic modulus of composites where the filler geometry is not considered and filler volume fraction is taken as the prime parameter [22, 24].The fitting of these models with the experimental data is shown in Fig. $3 \mathrm{~d}$. The Halpin-Tsai model and Hui-Shia model have predicted the values very closely. Halpin-Tsai model predicts the modulus values at different aspect ratios where average value of aspect ratio is taken. Hence, this model gives the close prediction for the elastic modulus. Hui-Shia model predicts the modulus values at an average aspect ratio of 12 , whereas the Halpin-Tsai fits at an average aspect ratio of 4 . The aspect ratio from the TEM images has been calculated as 20 . The difference in the aspect ratio for both fitting models and practical value is due to the fact that the formation of both the model is based on different assumptions. Halpin-Tsai model takes the random dispersion of the particles, but it does not consider the filler particles interaction with the matrix. On the other hand, Hui-Shia model takes the assumption of the unidirectionally oriented particles as well as the perfect interfacial bonding between the filler and the matrix. However, the practical arrangement is very different. The filler particles do not have a constant geometry throughout the composite but instead have random distribution of shapes and sizes. Some filler particles are exfoliated in the matrix, whereas most are intercalated. There are particles which get agglomerated also. In all these conditions, the aspect ratio gets changed. There are interfacial interactions present between the matrix and filler particles due to which property enhancement is affected to a great extent. The impurities and the microvoids are also present in the matrix. All of these factors contribute to the alteration of the experimental results and thus are different from what is predicted theoretically. Voigt upper bound [24] and Reuss lower bound [22] models have shown the upper and lower limits of modulus prediction. The experimental values and predicted values are lying between the two limits. However, the improvement in mechanical properties is well predicted through the Halpin-Tsai and Hui-Shia models at the aspect ratio values of 4 and 12, respectively. Hence, both the micromechanical models predict the values closely for the systems presented and are suitable for this system.

\subsection{Microhardness of nanohybrids}

The hardness of the samples has been found out through Vickers hardness test. The indentations on pure PET and P-B nanohybrids are shown in Fig. 3e. The nanohybrids have clay particles hence showing comparatively darker imprints. The hardness values have been increasing for the increased filler percentage which is shown in Fig. $3 f$ (experimental curve). The clay particles induce stiffness in the polymer matrix which is the reason of the increased hardness of the nanohybrids. The dispersed nanoclay particles form a network structure so that better stress transfer occurs. Moreover, the interactions and interfacial bonding between nanoclay particles and polymer matrix also play a role in increased hardness of the nanohybrids. The hardness is also linearly dependent on the Young's modulus of the material and as the modulus has been increasing continuously with the filler percentage, the microhardness has also been following the same behavior [25]. In previous reported values, the filler hardness was 83 times the matrix hardness and the increment in nanocomposite hardness is 
$14 \%$ for 7 volume percentage of the filler $\left(\mathrm{Al}_{2} \mathrm{O}_{3}\right)$ [27]. Here in this work, the filler hardness is very less, i.e., 1.28 times the matrix hardness, and the hardness increment achieved is $16 \%$ for 6 volume percentages of the filler. The hardness achievement, in spite of the fact that the filler hardness is not very high, is due to the reason that the filler has large aspect ratio. The clay platelets form structure and induce stiffness in the matrix causing greater hardness. The prediction of the hardness values has been done by the two models named MROM (Modified Rule of Mixtures) [26] and Halpin-Tsai model [27].

\subsubsection{MROM (modified rule of mixture)}

Hardness of composite has been predicted by MROM which is defined as:

$H_{c}=\beta H_{f} \phi_{f}+H_{m} \phi_{m}$

where $H_{c^{\prime}} H_{f}$ and $H_{m}$ are the hardness of the composite, filler and matrix. $\beta$ is the fitting parameter also called strengthening efficiency factor, which depends on the filler geometry, aspect ratio and filler particle reinforcements in the polymer matrix. $\phi_{f}$ and $\phi_{m}$ are the volume fractions for filler and matrix, respectively.

\subsubsection{Modified Halpin-Tsai model}

This model takes into account the random orientation of the filler as well as different shapes and types of filler materials. The modulus term has been replaced by the hardness in conventional Halpin-Tsai equation and proposed as:

$H_{c}=H_{m}\left[\frac{1+\varsigma \eta V_{f}}{1-\eta V_{f}}\right]$

$\eta=\frac{\frac{H_{f}}{H_{m}}-1}{\frac{H_{f}}{H_{m}}+\varsigma}$

here $\varsigma$ is an adjustable fitting parameter. The upper bound and lower bound can be found by putting $\varsigma$ as infinite and 0 , respectively. $\varsigma$ depends on the geometry, loading direction and the packing as well as the orientation of the filler particles. The values of matrix hardness have been taken as 11.54VHN and nanoclay hardness as $14.88 \mathrm{VHN}$ [26]. The MROM predicts the values very closely at a fitting parameter of 0.8 . The value of fitting parameter varies proportional to the aspect ratio [28]. Here, the higher value of fitting parameter as compared to previous reported value [27] is due to the higher value of aspect ratio and lower difference in hardness of polymer matrix and filler. Halpin-Tsai model under-predicts the hardness values. The reason behind this could be the variation in aspect ratio as well as lower difference in hardness values of polymer matrix and filler. Hence, the MROM model is best suited for the prediction of the hardness of the PET nanohybrids at a fitting parameter of 0.8 .

\subsubsection{Analysis of stress distribution}

The distribution of stress in the matrix in the presence of nanoparticles has been shown by ANSYS software. The dimensions of the two-dimensional polymer plate have been taken as $500 \times 500 \mathrm{~nm}^{2}$, and the dimensions of dispersed nanoparticles have been taken as length $\sim 100 \mathrm{~nm}$ and thickness of $10 \mathrm{~nm}$. The nanoparticles have been shown to be dispersed randomly at different angles. The properties as input are given as: for polymer matrix: Young's modulus $=1.51 \mathrm{GPa}$, Poisson's ratio $=0.33$; for nanoparticles: Young's modulus $=170 \mathrm{GPa}$, Poisson's ratio $=0.23$. The nanoparticle and matrix interface has been taken as perfectly bonded. The bottom part of the plate has been fixed, and tensile force has been applied on the upper part. Pure PET and its nanohybrids' output on applying tensile force are shown in Fig. 4a, b, respectively, where the values shown are indicative not exact. Pure PET has shown the stress distributed uniformly in the matrix. The concentrated stress distribution in the corners is due to the fixing of the plate from the bottom. When the nanoparticles have been dispersed in the matrix and the tensile force is applied, the stress distribution has been changed. Most of the stress has been taken up by the nanoparticles, and the stress distributed in the matrix has been reduced. The different geometries and alignments of the nanoparticle in the matrix are shown in the supplementary Figure S1-S4. The nanoparticle aligned parallel in the direction of applied load has been taken up the maximum amount of stress. The stress in the matrix beside nanoparticle (aligned perpendicular to the loading direction) has been reduced to minimum. The stress has been distributed above and below the nanoparticle. This shows that the particles aligned in the direction of applied load bear the maximum amount of stress. The particles aligned perpendicular to the direction of applied load bear the same load as the matrix through their length and have concentrated stress at their ends. Overall, the stress borne by the matrix has been reduced in the presence of the nanoparticles. The particles aligned in the direction of applied load have borne the maximum stress as shown in Fig. $4 \mathrm{~b}$.

\subsection{Effect of stretching on structure}

The effect of stretching has been observed through SAXS and wide-angle XRD studies. The SAXS images of unstretched and stretched samples are shown in Fig. 5a. 
(a)
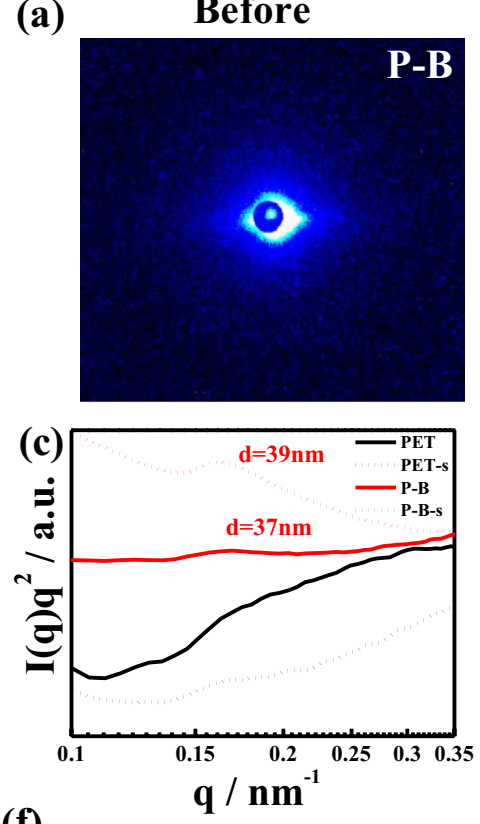

(f)

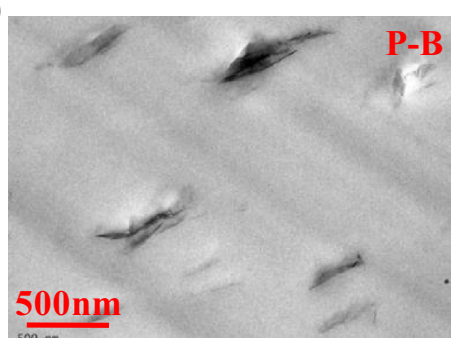

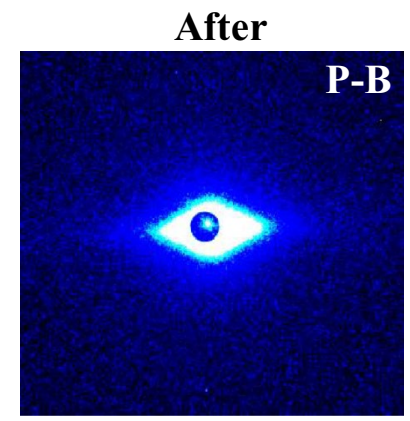
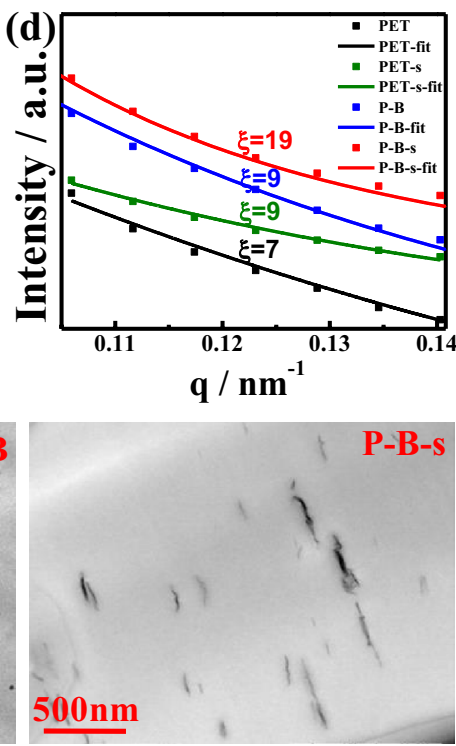

(b)
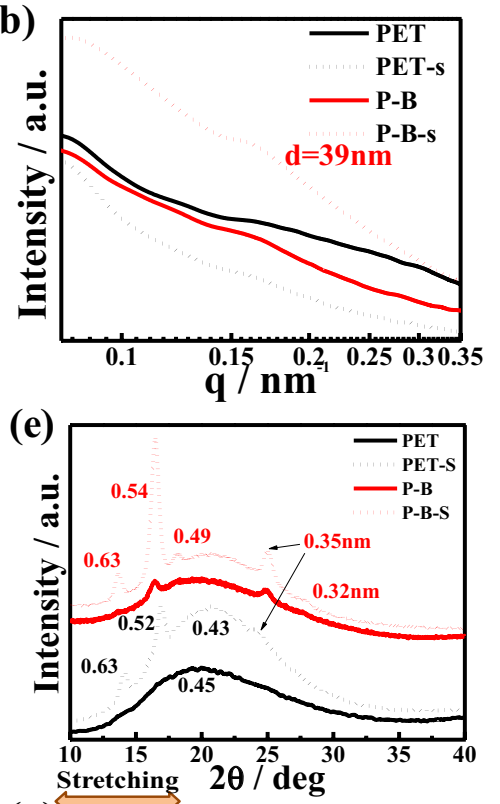

(g)

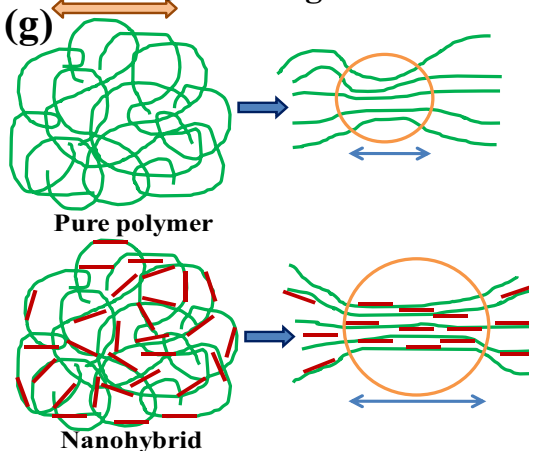

Fig. 5 a SAXS images of unstretched and stretched samples of PET and $\mathrm{P}-\mathrm{B} ; \mathbf{b}$ intensity versus wave vector plot extracted from the SAXS images; c Lorentz corrected profiles of the unstretched and stretched samples; $\mathbf{d}$ Debye-Bueche model fitting for calculation of

The unstretched samples have shown a uniform ring because of the uniform distribution of the nanoclay in the nanohybrid. Upon stretching the samples, the SAXS patterns have shown a streak occurring perpendicular to the stretching direction. This is the result of the partial orientation of clay particles and short-range ordering in the polymer matrix. The streak can also be the result of the elongation of voids present in the matrix [29]. The formation of the different mesophases has been reported previously [29-31]. Intensity $(/(q))$ versus scattering vector $(q)$ is plotted in Fig. $5 b$. The peak appears at $39 \mathrm{~nm}$ which is more evident in the Lorentz corrected profile $\left[/(q) \cdot q^{2} \mathrm{vs}\right.$. $q$ plot] in Fig. $5 \mathrm{c}$. The peak shows the characteristic length of $37 \mathrm{~nm}$ in unstretched P-B which has been increased to $39 \mathrm{~nm}$ in the stretched nanohybrid. The increase in the characteristic peak is due to the short-range ordering and the partial alignment of the clay particles in the stretching direction. The initial linear part has been fitted using correlation lengths of unstretched and stretched samples; e wideangle XRD plots of unstretched and stretched samples; and $\mathbf{f}$ schematic diagram of the structural advancement upon stretching in PET and P-B nanohybrids

Debye-Bueche model $[33,34]$ to obtain the correlation length of the samples $\left[/(q)=A /\left(1+\xi^{2} q^{2}\right)^{2}\right.$, where $A$ is constant and $\xi$ is termed as correlation length of the blob] (Fig. $5 \mathrm{~d}$ ). The correlation lengths of the blob have found to be increasing upon stretching the sample. The blob size is further increasing in the presence of nanoclay. XRD of the unstretched and stretched samples are shown in Fig. 5e. The unstretched sample of PET has shown a hump having peak at $0.45 \mathrm{~nm}$ which signifies the amorphous structure of the polymer matrix (texturing). On stretching the sample, the hump has shifted to $0.43 \mathrm{~nm}$. The shifting is due to long-range ordering arising from the interaction of nanoclay with the polymer matrix. The stretched sample has shown two peaks which appear at 0.52 and $0.63 \mathrm{~nm}$. The peak at $0.52 \mathrm{~nm}$ corresponds to (010), whereas the peak at $0.63 \mathrm{~nm}$ appears due to some local structure formations $[31,32]$. XRD spectra of unstretched P-B have shown some characteristic peaks of $30 \mathrm{~B}$ nanoclay at 0.54 and $0.35 \mathrm{~nm}$. 
XRD spectra of 30B clay are given in Supplementary Fig. S5. On stretching the nanohybrid, the intensities of the characteristic peaks have increased and few more characteristic peaks have also appeared ( 0.49 and $0.32 \mathrm{~nm})$. The increased intensity is due to the induced coherency in the molecular structure because of the presence of nanoclay. The average aspect ratio of the nanoclay particles has also been increased from 20 to 47 on stretching as shown in Fig. 5f. The nanoclay particles tend to align the molecular chains more and hence increase the blob size. The clay platelets between the polymeric chains align themselves as well as the polymeric chains. The effect of stretching on the molecular structure is shown in the schematic Fig. $5 \mathrm{~g}$, where blob size has been shown increasing on stretching the nanohybrid. However, the mechanical strength has improved in PET nanohybrid significantly without any considerable loss of toughness as compared to literature reports. The dispersion of nanoclay and nanostructural arrangement under uniaxial stretching is responsible for those properties development as opposed to degradation in melt processed nanohybrid.

\section{Conclusion}

PET nanohybrids have been prepared through solution casting route. The dispersion of nanoclay has been tested through TEM and XRD and found to be homogeneous and uniform. The interactions of nanoclay with PET matrix have been observed using FTIR. Thermal properties of nanohybrids have been tested by TGA and DSC where the degradation temperature and glass transition temperature are found to be unchanged. The nanohybrids have shown significant increment in tensile properties, and the modulus has been increased up to $93 \%$ for 8 wt $\%$ filler concentration. Toughness has been slightly compromised as opposed to huge loss in literature reports. The optimum value for the modulus and toughness has been obtained at $4 \%$ filler concentration where modulus has been increased $21 \%$ and toughness reduction has been $12 \%$ only. Hardness of the nanohybrids has been tested by Vickers hardness test where the hardness has increased up to $16 \%$ for 8 wt $\%$ of filler concentration in nanohybrid. The analysis of stress distribution in the presence of nanoclay has been done using ANSYS software. The clay particles have been found to bear most of the stress in nanohybrids. Effect of stretching on the structure has been studied using SAXS of pure PET and PET nanohybrid. The presence of clay in the polymer matrix has increased the local structuring/ ordering which has also been reflected in the wide-angle XRD patterns. The method proposed results in retaining toughness which is important for the material to be used in various applications. The effect of geometry of fillers on the stress distribution has been studied so as to impart the light on the behavior of nanofillers in different orientations. The stretching behaviors of nanohybrids have been studied to observe the effect of nanoclay in PET matrix. The PET nanohybrids have been found to have practical usage due to improved properties.

Acknowledgements DS acknowledges the institute for her teaching assistantship.

\section{Compliance with ethical standards}

Conflict of interest Authors declare no conflict of interest.

\section{References}

1. Yousfi M, Soulestin J, Vergnes B, Lacrampe MF, Krawczak P (2013) Morphology and mechanical properties of PET/PE blends compatibilized by nanoclays: effect of thermal stability of nanofiller organic modifier. J Appl Polym Sci 128(5):2766-2778

2. Nguyen QT, Baird DG (2006) Preparation of polymer-clay nanohybrids and their properties. Adv Polym Technol 25(4):270-285

3. Kapusetti G, Misra N, Singh V, Srivastava S, Roy P, Dana K, Maiti $P(2014)$ Bone cement based nanohybrid as a super biomaterial for bone healing. J Mater Chem B 2(25):3984-3997

4. Jana KK, Charan C, Shahi VK, Mitra K, Ray B, Rana D, Maiti P (2015) Functionalized poly(vinylidene fluoride) nanohybrid for superior fuel cell membrane. J Membr Sci 481:124-136

5. Nam PH, Maiti P, Okamoto M, Kotaka T, Hasegawa N, Usuki $A$ (2001) A hierarchical structure and properties of intercalated polypropylene/clay nanohybrids. Polymer 42(23):9633-9640

6. Pandey S, Jana KK, Aswal VK, Rana D, Maiti P (2017) Effect of nanoparticle on the mechanical and gas barrier properties of thermoplastic polyurethane. Appl Clay Sci 146:468-474

7. Ke Z, Yongping B (2005) Improve the gas barrier property of PET film with montmorillonite by in situ interlayer polymerization. Mater Lett 59(27):3348-3351

8. Frounchi M, Dourbash A (2009) Oxygen barrier properties of poly (ethylene terephthalate) nanohybrid films. Macromol Mater Eng 294(1):68-74

9. Lee WD, Im SS, Lim HM, Kim KJ (2006) Preparation and properties of layered double hydroxide/poly (ethylene terephthalate) nanohybrids by direct melt compounding. Polymer 47(4):1364-1371

10. Vassiliou AA, Chrissafis K, Bikiaris DN (2010) In situ prepared PET nanohybrids: effect of organically modified montmorillonite and fumed silica nanoparticles on PET physical properties and thermal degradation kinetics. ThermochimicaActa 500(1-2):21-29

11. Shen $Y$, Harkin-Jones $E$, Hornsby $P$, McNally $T$, Abu-Zurayk $R$ (2011) The effect of temperature and strain rate on the deformation behaviour, structure development and properties of biaxially stretched PET-clay nanohybrids. Compos Sci Technol 71(5):758-764

12. Zeng Z, Matuschek D, Studer A, Schwickert C, Pöttgen R, Eckert $\mathrm{H}$ (2013) Synthesis and characterization of inorganic-organic hybrid materials based on the intercalation of stable organic radicals into a fluoromica clay. Dalton Trans 42(24):8585-8596

13. Lin Y, Tyler R, Sun H, Shi K, Schiraldi DA (2017) Improving oxygen barrier property of biaxially oriented PET/phosphate glass composite films. Polymer 127:236-240 
14. Ghasemi H, Carreau PJ, Kamal MR, Tabatabaei SH (2012) Properties of PET/clay nanohybrid films. Polym Eng Sci 52(2):420-430

15. Ghanbari A, Heuzey MC, Carreau PJ, Ton-That MT (2013) A novel approach to control thermal degradation of PET/organoclay nanocomposites and improve clay exfoliation. Polymer 54(4):1361-1369

16. Scaffaro R, Botta L, Ceraulo M, La Mantia FP (2011) Effect of kind and content of organo modified clay on properties of PET nanohybrids. J Appl Polym Sci 122(1):384-392

17. Yang F, Mubarak C, Keiegel R, Kannan RM (2017) Supercritical carbon dioxide ( $\mathrm{scCO} 2)$ dispersion of poly (ethylene terephthalate)/clay nanocomposites: structural, mechanical, thermal, and barrier properties. J Appl Polym Sci. https://doi.org/10.1002/ app.44779

18. Tiwari VK, Prasad AK, Singh V, Jana KK, Misra M, Prasad CD, Maiti $P$ (2013) Nanoparticle and process induced super toughened piezoelectric hybrid materials: the effect of stretching on filled system. Macromolecules 46(14):5595-5603

19. Nand AV, Ray S, Travas-Sejdic J, Kilmartin PA (2012) Characterization of polyethylene terephthalate/polyaniline blends as potential antioxidant materials. Mater Chem Phys 134(1):443-450

20. Djebara M, Stoquert JP, Abdesselam M, Muller D, Chami AC (2012) FTIR analysis of polyethylene terephthalate irradiated by MeV He+. Nucl Instrum Methods Phys Res Sect B 274:70-77

21. Hui CY, Shia D (1998) Simple formulae for the effective moduli of unidirectional aligned composites. Polym Eng Sci 38(5):774-782

22. Affdl JC, Kardos JL (1976) The Halpin-Tsai equations: A review. Polym Eng Sci 16(5):344-352

23. Dong Y, Bhattacharyya D (2010) Morphological-image analysis based numerical modelling of organoclay filled nanocomposites. Mech Adv Mater Struct 17(7):534-541

24. Hu H, Onyebueke L, Abatan A (2010) Characterizing and modeling mechanical properties of nanocomposites-review and evaluation. J Miner Mater Charact Eng 9(04):275

25. Calleja FB, Fakirov S (2007) Microhardness of polymers. Cambridge University Press, Cambridge
26. Minkova L, Peneva Y, Valcheva M, Filippi S, Pracella M, Anguillesi I, Magagnini P (2010) Morphology, microhardness, and flammability of compatibilized polyethylene/clay nanocomposites. Polym Eng Sci 50(7):1306-1314

27. Goyal RK, Tiwari AN, Negi YS (2008) Microhardness of PEEK/ ceramic micro-and nanocomposites: correlation with HalpinTsai model. Mater Sci Eng A 491(1-2):230-236

28. Kuo MC, Tsai CM, Huang JC, Chen M (2005) PEEK composites reinforced by nano-sized $\mathrm{SiO}_{2}$ and $\mathrm{Al}_{2} \mathrm{O}_{3}$ particulates. Mater Chem Phys 90(1):185-195

29. Todorov LV, Martins Cl, Viana JC (2013) In situ WAXS/SAXS structural evolution study during uniaxial stretching of poly (ethylene therephthalate) nanocomposites in solid state: poly (ethylene therephthalate)/montmorillonite nanocomposites. J Appl Polym Sci 128(5):2884-2895

30. Kawakami D, Hsiao BS, Burger C, Ran S, Avila-Orta C, Sics I, Kikutani T, Jacob Kl, Chu B (2005) Deformation-induced phase transition and superstructure formation in poly (ethylene terephthalate). Macromolecules 38(1):91-103

31. Kawakami D, Ran S, Burger C, Fu B, Sics I, Chu B, Hsiao BS (2003) Mechanism of structural formation by uniaxial deformation in amorphous poly(ethylene terephthalate) above the glass temperature. Macromolecules 36(25):9275-9280

32. Ran S, Wang Z, Burger C, Chu B, Hsiao BS (2002) Mesophase as the precursor for strain-induced crystallization in amorphous poly(ethylene terephthalate) film. Macromolecules 35(27):10102-10107

33. Debye P, Bueche AM (1949) Scattering by an inhomogeneous solid. J Appl Phys 20(6):518-525

34. Mishra A, Aswal VK, Maiti P (2010) Nanostructure to microstructure self-assembly of aliphatic polyurethanes: the effect on mechanical properties. J Phys Chem B 114(16):5292-5300

Publisher's Note Springer Nature remains neutral with regard to jurisdictional claims in published maps and institutional affiliations. 